\title{
A ISABEL MORANT EN SU JUBILACIÓN DESDE LA AMISTAD
}

\author{
Carmen Aranegui Gascó \\ Universitat de València
}

El decanato de la Facultad de Geografía e Historia me ha encomendado que destaque la trayectoria de la Prof. Isabel Morant Deusa en este acto dedicado a los compañeros que se han jubilado en 2017. He aceptado con gusto, contando con que se me disculpará la falta de rigor al valorar sus aportaciones académicas a la Historia Moderna, puesto que mi especialidad es muy distinta a la suya.

Mediante una aproximación desde la amistad, haré referencia, en primer lugar, a su curriculum vitae, porque pienso que este acto es una buena ocasión para relacionar su trabajo, desde mi perspectiva, con los avances de esta facultad protagonizados por una minoría que se formó en ella hacia el final de los años 1970 y el comienzo de los 80 y obtuvo después, afortunadamente, una plaza de profesor no numerario en la misma. Se ha reconocido la vanguardia del manual de Ubieto-Reglá-Jover-Seco ${ }^{1}$ que se gestó aquí, y que queda atrás, pero no se ha reconocido la aportación de una generación posterior, muy valiosa.

Algunos de aquellos estudiantes de los 70 y 80 tuvieron un profesorado apenas cinco o seis años mayor que ellos, que les inculcó desde la cercanía el impulso de movilización y competencia que se vivía entonces respecto a todo, también en lo relativo a los -ismos de la investigación histórica (historicismo, positivismo, estructuralismo, nacionalismo, marxismo...), que se sucedían con rapidez en los círculos progresistas, porque se ponían en tela de juicio cada poco tiempo. Creo que en el departamento de Historia Moderna Sebastián García Martínez (1942-1986) y Manuel Ardit (1941-2013) representaron aquel objetivo de actualizar métodos y contenidos para hacer una Historia Económica y Social crítica, conscientes de que era preciso salir de las torres de marfil académicas. Y justo después cabe situar la ampliación de contenidos en la que participó Isabel Morant.

Se doctoró en 1984 con una tesis entonces al uso, es decir, sobre el País Valenciano, como la inmensa mayoría de las que se defendían entonces, y con

1 A. Ubieto; J. Reglá; J.M. Jover; C. Seco (1969): Introducción a la Historia de España, Barcelona: Teide. 
un enfoque económico y social, titulada "El declive del señorío: los dominios del Ducado de Gandia: 1705-1837"'2, publicada como un estudio de caso, importante para dar a conocer la singularidad valenciana.

Ese mismo año su dedicación cambió, puesto que pasó a formar parte del equipo del Rector electo Ramon Lapiedra, como primera Vicerrectora del primer gobierno democrático, nacionalista y de izquierdas, de la Universitat de València. Antes de esa fecha, cuando el Rector-siempre de Medicina o Derecho, las dos facultades decisivas en esta universidad-cambiaba, se decía que lo habían nombrado o que lo había puesto porque los cargos públicos eran cuestión de decisiones no ponderadas por un electorado, que en el caso de Lapiedra sí que estuvo avalado por una representación de más de cuatrocientos claustrales, frente al candidato Aurelio Martínez, del PSPV.

Isabel Morant fue una eficiente Vicerrectora de Cultura cooptada por los estudiantes nacionalistas del BEA, de cuya gestión quedan, al menos, dos infraestructuras importantes: el Servei de Publicacions (1986) y el Campo de Deportes de Blasco Ibáñez, instalado sobre solares muy cotizados de la ciudad, además de una contribución, desde 1986, al debate cultural y político proyectada al gran público por la Universitat d'Estiu de Gandia, en colaboración con el ayuntamiento de esta ciudad, que aquellos años invitó a quienes mantenían retos de innovación en diversos campos.

En 1990 dejó su cargo en el Rectorado y aprovechó el plazo reglamentario de readaptación a las tareas docentes para realizar una estancia en París que le permitió poner en práctica algo que seguramente ya tenía asumido, como era la necesidad de aprender fuera de España cómo abordar una temática hasta entonces ausente en nuestros títulos universitarios, como era la Historia de las Mujeres, que con Isabel Morant se introdujo en esta facultad. Cuando Georges Duby y Michelle Perrot abordaron la cuestión en una obra colectiva, publicada en cinco volúmenes ${ }^{3}$, en la edición francesa no había ninguna contribución española, por lo que tuvo que añadirse un pequeño apéndice 'español' cuando Taurus tradujo aquella publicación.

En l'École de Hautes Études en Sciences Sociales Isabel Morant constató que las fuentes de la historia no se encontraban solo en los archivos sino que, para equilibrar el androcentrismo de la Historia, que era lo que perseguía, era necesario salir de aquellos y recurrir a todo lo que pudiera mostrar el marco relacional de las vidas de las mujeres: sus contextos sociales y culturales, si-

\footnotetext{
2 I. Morant (1984): El declive del señorío: los dominios del ducado de Gandia 17051837, Institució Alfons el Magnànim,Valencia.

${ }^{3}$ G. Duby; M. Perrot (dirs.) (1991-1992): Histoire des femmes en occident, 5 vols., París: Pion.
} 
guiendo una metodología adecuada, como la aplicada a la historización de la vida privada ${ }^{4}$, que exigía el conocimiento del contexto de las fuentes utilizadas y del marco histórico en que se abordaba cada problemática, los siglos XVII, XVIII y parte del XIX en su caso.

Fue de este modo como cristalizó la colección Feminismos en 1991, como una coedición principalmente de Ediciones Cátedra, el Instituto de la Mujer (hasta 2013) y la Universitat de València, que puso al alcance de un público no exclusivamente universitario textos, generalmente breves, relacionados con una diversidad de contribuciones a la Historia de las Mujeres, que siguen publicándose con éxito de ventas hasta la actualidad. Este es un mérito relevante de Isabel Morant.

En este ambiente hay que situar las primeras tesis doctorales defendidas en esta facultad sobre Historia de las mujeres, dirigidas por Isabel Morant. Entre las primeras doctoras que yo recuerde se cuentan Dolores Sánchez Durá (1993), Mónica Bolufer, que continúa impartiendo Historia de las mujeres en nuestras aulas, y Estrella Garrido, que dejó la universidad, a las que han seguido muchas otras.

Es de resaltar que, además, la ampliación de estos marcos de estudio nos sirvió al resto del profesorado, porque pudimos asistir a conferencias y a seminarios, impartidos en Valencia por reconocidos especialistas en temas nuevos, que Isabel influyó para que vinieran a Valencia.

Y fue de este modo como, de acuerdo con la historia de la lectura de Roger Chartier, historiador de la cuarta generación de la Escuela de Annales; en contacto con Geneviéve Fraisse, política y filósofa feminista de izquierdas, y con Luisa Accati, historiadora y antropóloga feminista de la Universidad de Trieste, y con muchas otras autoras, entre las que deseo mencionar a Sonia Mattalia, catedrática de Literatura Latinoamericana, que falleció en 2012, Isabel se orientó hacia unas determinadas fuentes, como el ensayo, el teatro, las biografías y la correspondencia de mujeres y hombres de la Ilustración, y comenzó a utilizar con gran rigor la novela sentimental como exponente del colectivo femenino, todo lo cual le ha llevado a trabajar no sobre la literatura sino sobre la historia de las relaciones sentimentales, del matrimonio y de la familia, sobre los que versan gran parte de sus publicaciones ${ }^{5}$.

\footnotetext{
${ }^{4}$ P. Ariès (ed.) (1985-1987): Histoire de la vie privée, 5 vols., París: Seuil.

5 I. Morant; M. Bolufer (1998): Amor, matrimonio y familia: la construcción histórica de la familia moderna, Madrid: Síntesis. I. Morant (2002): Discursos de la vida buena: Matrimonio, mujer y sexualidad en la literatura humanista, col Feminismos, Madrid: Cátedra.
} 
Ante una pregunta sobre estas realizada en Buenos Aires en 2007, Isabel responde:

...Tengo dos libros sobre ese tema. Uno se llama Amor, matrimonio y familia (1998), dedicado al matrimonio en el XVIII. Luego, volví un poco hacia atrás, pues me fascinó la mirada de los humanistas frente al matrimonio. Me di cuenta que se podía trabajar muy bien con el discurso humanista y escribí sobre matrimonio y sexualidad en la literatura europea Discursos de la vida buena (2002).

En 2005-2006 la edición del gran proyecto que ha mostrado la vitalidad de la historia feminista de las mujeres en España vio la luz bajo la dirección de nuestra compañera y la asistencia de Mónica Bolufer. Reunieron textos de un centenar de autores españoles y latino-americanos, predominantemente mujeres, que recorre todo el ciclo histórico (incluida la prehistoria) . $^{6}$

Por todo esto y por su versatilidad Isabel Morant es una mujer reconocida públicamente: por el grupo Mujeres Universitarias de los años 1970. En 1986 y 1987 por el Seminari Interdisciplinar d'Investigació Feminista y, finalmente, por el Institut Universitari d'Estudis de la Dona (1991 y 1994), en lo que a la Universitat de València corresponde.

Fuera de esta, ha pertenecido al Consell Valencià de Cultura (2004-2011), ha sido premiada por la promoción de la igualdad en el conocimiento (2007) y por la FECYT (Fundación Española para la Ciencia y la Tecnología) en 2016. Y, finalmente, acaba de merecer la medalla de oro de la Universitat de València.

La segunda referencia de Isabel Morant que deseo destacar se refiere a su vertiente política en apoyo de la igualdad entre hombre y mujeres, que creo que también se reforzó en nuestra colega profesora emérita mediante el diálogo con mujeres de fuera de la facultad y de fuera de España.

Cuando Alessandra Bocchetti ${ }^{7}$, que conocimos en Roma a comienzos de los años 1980 por mediación de Rafael Valls, escribió:

Quiero mejorar la vida de las mujeres porque solo así mejorará mi propia vida.

Expresó una consigna política que carga el mensaje del lado de la solidaridad entre las mujeres. Y esta es una consigna compartida por Isabel Morant.

${ }^{6}$ I. Morant, coord. (2005-2006): Historia de las mujeres en España y en América Latina, 4 vols., col Feminismos, Madrid: Cátedra.

7 A. Bocchetti (1999): Lo que quiere una mujer, col. Feminismos, Madrid: Cátedra. 
Por otra parte, la discriminación por razones de sexo ha insistido, con más frecuencia que en la solidaridad, en la denuncia, basada en datos sociológicos, que también es necesaria, qué duda cabe.

El compromiso feminista de Isabel Morant creo que contiene solidaridad y denuncia, y que, sobre todo, se vehicula a través del conocimiento que le han proporcionado sus estudios, vertidos hacia su docencia y sus espacios de comunicación (conferencias y artículos de prensa).

La siguiente manifestación es ilustrativa de sus objetivos:

"Me gusta pensar que hay -o que habrá- un público lector que, en las cuestiones desveladas por la historia de las mujeres, reconozca un saber nuevo y más vital sobre nosotros mismos, sobre nuestras vidas y nuestras relaciones con los demás, un público que pueda servirse de la historia, pensar las vidas que vivimos y vislumbrar, si cabe, la vida que queremos".

En las fuentes de su compromiso están Virginia Woolf, El Segundo Sexo, Rousseau y muchos pensadores, políticos y ensayistas del siglo XVIII.

Pero a mi entender, y hasta el momento, las ochenta páginas que constituyen el prólogo del Discurso sobre la felicidad de Mme. du Châtelet ${ }^{8}$ reflejan lo que podríamos llamar el decálogo, en sentido figurado, de un modelo en el que tienen cabida mujeres inteligentes y dueñas de sí mismas, visto a través de la relación entre una noble científica y un insigne filósofo, Voltaire, que vivieron en plena Ilustración una intensa relación amorosa mientras defendían la superioridad de la razón. La cita de Voltaire elegida para el inicio de dicho estudio, recuerda que quien se desentiende del amor no es ni feliz ni sabio. Sigue un comentario de la correspondencia de una mujer noble del París de mediados del siglo XVIII a quien la instrucción que recibió le dio alas con las que supo situarse a la altura intelectual de los hombres de su entorno. Una mujer que exaltó la pasión amorosa y el amor al estudio sobre el que señaló:

Es seguro que el amor al estudio es bastante menos necesario para la felicidad de los hombres que para la de las mujeres. Los hombres...tienen otros medios de alcanzar la gloria... ... pero las mujeres... ... solo les queda el estudio para consolarse de todas las exclusiones y de todas las dependencias a las que se encuentran condenadas...

Isabel Morant se ha esforzado en, según sus propias palabras, "instruir deleitando" y en hacer una investigación que nos ayuda a comprender por qué hay sexismo en la Historia.

${ }^{8}$ I. Morant (ed.) (1996): Madame du Châtelet. Discurso sobre la felicidad y Correspondencia, Col. Feminismos, Madrid: Cátedra. 
Estoy segura de que su vida profesional y política no ha terminado. Tiene mucho que ofrecernos todavía, pues su vocación y pasión por la historia relacional en que aparece el colectivo femenino sigue viva.

Y, además, seguiremos pidiéndole que nos 'instruya deleitando'.

¡Bienvenida, querida amiga, a una etapa gozosa de la vida! 